\title{
Pore characterization through propagator-resolved transverse relaxation exchange
}

\author{
K. E. Washburn, ${ }^{1}$ C. H. Arns, ${ }^{2}$ and P. T. Callaghan ${ }^{1}$ \\ ${ }^{1}$ School of Chemical and Physical Sciences, Victoria University of Wellington, Wellington, New Zealand \\ ${ }^{2}$ Department of Applied Mathematics, Research School of Physical Sciences and Engineering, \\ Australian National University, Canberra ACT 0200, Australia
}

(Received 23 January 2008; published 8 May 2008)

\begin{abstract}
We use the propagator-resolved transverse relaxation exchange experiment to characterize the pore space and fluid behavior of water saturated, tight-packed quartz sand. The experiment uses $T_{2}$ exchange plots to observe the number of molecules that shift their environment for a range of mixing times. The propagator dimension allows us to determine how far the molecules have moved. The peak intensities are integrated and then plotted as a function of displacement and mixing time. We also model our system using both a probabilistic pore-hopping simulation and a spreading Gaussian model. We use the results of these simulations to interpret the peak intensity plots. From this, we can estimate pore features such as characteristic time, pore radii, and interpore spacing. The tortuosity of the different pore sizes can then be calculated from these values.

DOI: 10.1103/PhysRevE.77.051203

PACS number(s): 47.56. $+\mathrm{r}, 81.05 . \mathrm{Rm}, 82.56 . \mathrm{Fk}, 82.56 . \mathrm{Lz}$
\end{abstract}

\section{INTRODUCTION}

Characterization of porous media and understanding the movement of the fluids within is important to a wide range of fields such as biology, oil exploration, and chemical engineering. Nuclear magnetic resonance (NMR) is useful in studying the pore space because it is noninvasive, can be used on opaque samples, and can be performed in situ. Pore size is known to be correlated with the transverse $\left(T_{2}\right)$ relaxation time, the rate of spin decoherence. In general, the smaller the pore, the more quickly the magnetization relaxes. This relation is described by the equation [1]

$$
\frac{1}{T_{2}}=\frac{1}{T_{2}^{\text {bulk }}}+\frac{1}{T_{2}^{\text {surface }}}=\frac{1}{T_{2}^{\text {bulk }}}+\rho \frac{S}{V},
$$

where $\rho$ is the surface relaxivity of the materials, $S$ is the pore surface area, $V$ is the volume of the pore, and $T_{2}^{\text {bulk }}$ is the bulk relaxation rate of the fluid. The transverse relaxation exchange experiment $[2,3]$ correlates the $T_{2}$ values of fluid molecules at two points in time that are separated by a mixing period in order to find how quickly fluid molecules move between pores of different sizes. If the $T_{2}$ value of a molecule changes during the mixing time, this is indicative, but not conclusive, that the fluid molecule has moved to a pore of another size.

A limitation of the $T_{2}-T_{2}$ exchange experiment is that it cannot differentiate between signals that come from spinbearing molecules remaining in their original pore and molecules that have diffused to a pore of similar size. In previous work, we demonstrated the technique of propagator-resolved transverse relaxation exchange spectroscopy [4], in which the NMR pulse sequence combined two inverse Laplace dimensions and a Fourier dimension. This method enhanced the transverse relaxation exchange experiment by using magnetic pulsed field gradients to measure the displacement of molecules [5] during the mixing time. A spatially dependent uniform linear magnetic gradient results in the spins experiencing a magnetic field $B_{0}+\mathbf{r} \cdot \nabla B$ where $B_{0}$ is the static magnetic field, $B$ is the component parallel to $B_{0}$ of a much smaller applied gradient field, and $\mathbf{r}$ is the location of the spin in the sample. Note that the magnetic field gradient is defined by $\mathbf{g}=\boldsymbol{\nabla} B$ and is designed to be of consistent strength and direction. Thus we may write the effective Larmor frequency

$$
\omega=\gamma B_{0}+\gamma \mathbf{r} \cdot \mathbf{g},
$$

where $\omega$ is the Larmor frequency and $\gamma$ is the gyromagnetic ratio.

The spin-bearing molecules migrate during the experimental delay time $\Delta$. After this period, a second gradient pulse is applied to refocus the magnetization. The phase shift will cancel for molecules that have not moved with respect to the gradient direction whereas a phase shift will remain for those molecules that have changed their location. The extent of the phase shift is dependent on the distance the molecules have migrated. In an ideal experiment the magnetic field gradients of strength $g=|\mathbf{g}|$ are applied for a sufficiently short duration $\delta$ such that the spin migration during $\delta$ is insignificant. In this case we may define a reciprocal space $q=\gamma \delta g$ and write the echo attenuation resulting from the residual phase shifts as $[5,6]$

$$
\frac{M(q)}{M(0)}=\langle\exp (i q Z)\rangle=\int_{-\infty}^{\infty} P(Z, \Delta) \exp (i q Z) d Z,
$$

where $Z$ is the displacement of molecules parallel to the magnetic field gradient direction, over the time $\Delta$ and $P(Z, \Delta)$ is the probability distribution of displacement. A Fourier transform of the echo attenuation with respect to $q$ will give the fluid propagator $P(Z, \Delta)$, the distribution of molecular displacements in the sample. In the case where the displacements are diffusive and described by an effective diffusion coefficient $D$ the fluid propagator $P(Z, \Delta)$ will be described by the Gaussian

$$
P(Z, \Delta)=(4 \pi D \Delta)^{-1 / 2} \exp \left(-\frac{Z^{2}}{4 D \Delta}\right) .
$$

In this paper, we further develop the propagator-resolved transverse relaxation exchange method in addition to simulations to show how this technique may be used to charac- 


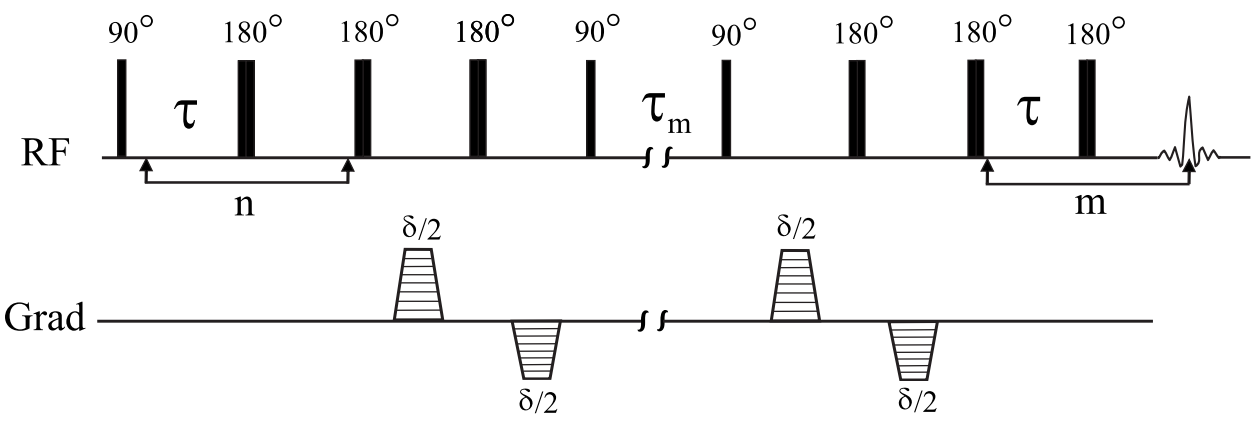

FIG. 1. Pulse sequence for the $T_{2}$ propagator-resolved exchange experiment terize the pore space as well as fluid dynamics. A significant feature of the current work is the presentation of a model that describes the response of the spin system. In particular, we present analytic expressions for the intensity of the peaks in the $T_{2}$ exchange spectra.

\section{EXPERIMENTAL}

\section{A. NMR}

The NMR experiments were carried out on a Bruker widebore $400 \mathrm{Mhz}$ Avance II spectrometer using microimaging gradients for the diffusion encoding. The sample consisted of tightly packed quartz sand saturated with distilled water. The sample was placed inside a $15 \mathrm{~mm}$ birdcage resonator and held at $25^{\circ} \mathrm{C}$ for the duration of experimentation.

\section{Internal gradient influence}

One concern was that different $T_{2}$ times in the data may arise not from differing pore size, but as an artifact of internal gradients [7] in the sample. As sand is known to sometimes contain paramagnetic impurities, this was a very real concern. We used two methods to satisfy ourselves that different $T_{2}$ values resulted from pore size differences and not internal gradients. One of the hallmarks of internal gradients is that the measured $T_{2}$ time may depend on the echo spacing of a Carr-Purcell-Meiboom Gill (CPMG) echo train [8,9]. We ran $T_{2}$-exchange experiments with echo spacing times of $70,150,300,400$, and $500 \mu \mathrm{s}$. Even up to $500 \mu \mathrm{s}$, peak placements in the spectra were virtually identical. While our experiments could have been performed with the shorter echo spacing of $70 \mu \mathrm{s}, 150 \mu \mathrm{s}$ is chosen here, as it appears adequate to compensate for the effects of internal gradients and encode for the shortest $T_{2}$ values while putting less strain on the duty cycle of the machine as well as minimizing the problem of coil heating.

We also ran experiments to measure the internal gradient strengths present in the sand sample. A forthcoming paper will discuss further details of this work. Our magnetic susceptibility difference is approximately $\Delta \chi \approx 30 \times 10^{-6}$ as measured by the inhomogeneous linewidth. As shown by Hürlimann [7], the maximum gradients in such a porous system are given by

$$
g_{\max } \approx\left(\frac{\gamma}{D_{0}}\right)^{1 / 2}\left(\Delta \chi B_{0}\right)^{3 / 2},
$$

where $D_{0}$ is the self-diffusion coefficient of the spin-bearing molecule, in our case water. For our sand system at a proton frequency of $400 \mathrm{MHz}$, Eq. (5) yields a value of $g_{\max } \approx 6.4$ $\times 10^{4} \mathrm{G} \mathrm{cm}^{-1}$. We then need to determine the length scale at which these gradients are relevant. $l^{*}$ is the internal gradient dephasing length, defined as

$$
l^{*}=\frac{\Delta \chi B_{0}}{g_{\max }} .
$$

This distance may be used to separate the system by pore size. Fluid molecules in pores smaller than the dephasing length will experience motionally averaged internal gradients while in pores larger than the dephasing length, we can assume a local gradient regime. For our sand system, this dephasing length is approximately $0.04 \mu \mathrm{m}$. As the pores we observe in our exchange experiments are much larger than this value, we expect that our echo dephasing is governed by the local gradient regime in which the local gradients are essentially static. Thus, while very large gradients do exist in the sample, the internal gradients for the pore length scales that we are concerned with are much smaller. For the $T_{2}$ values used in our exchange experiments, our measured internal gradient strengths are on the order of $100 \mathrm{G} \mathrm{cm}^{-1}$, whose influence can be avoided with the $150 \mu$ s echo spacing.

\section{Pulse sequence}

After the initial $90^{\circ}$ excitation pulse, the experiment begins with a $T_{2}$ encoding interval. To avoid influence from internal gradients, we measure the $T_{2}$ by keeping our echo spacing fixed at $150 \mu \mathrm{s}$, and varying the number of $180^{\circ}$ refocusing pulses (Fig. 1). We use only an even number of echoes to avoid the odd-even echo effect [10]. After the first $T_{2}$ encode, we apply the gradients for the diffusion measurement, using bipolar-pulsed field gradients sandwiched with a $180^{\circ}$ refocusing $\mathrm{rf}$ pulse to prevent possible interaction of internal gradients with the diffusion measurement [11]. The maximum applied gradients were $99 \mathrm{G} \mathrm{cm}^{-1}$. A subsequent $90^{\circ}$ pulse stores the magnetization along the $z$ axis for the mixing interval, where it does not experience $T_{2}$ relaxation, but only the longer $T_{1}$ relaxation. We used mixing times ranging from 50 to $550 \mathrm{~ms}$ in increments of $50 \mathrm{~ms}$. Unfortunately, mixing times shorter than this were not possible due to the limit of our gradient strength. After the mixing interval, another $90^{\circ}$ pulse returns the magnetization to the transverse plane. The complementing pair of bipolar gradients completes the measurement of the molecular diffusion dur- 
ing the mixing time. Finally, we perform our second $T_{2}$ encode simultaneously as acquisition, sampling the top of each echo in the CPMG train.

For our experiments, we acquired $4096 \times 66$ points in the $T_{2}$ dimensions and 32 points in the diffusion dimension. Each experiment required approximately $7 \mathrm{~h}$ to complete. Our phase cycling ensured that all the measured signal derived solely from the first, initial excitation pulse. The threedimensional (3D) data was zero filled to 64 points for fast Fourier transform (FFT), the equivalent of smooth interpolation in the spatial domain and then Fourier transformed along the diffusion axis to give the diffusion propagator. Planes of $T_{2}-T_{2}$ exchange data were taken from along the propagator. For each plane, we select the " $m$ " encoding times that corresponded to the " $n$ " encoding times used to produce a symmetric $62 \times 62$ matrix. The first four acquired points are not used due to oscillatory transients in the signal decay. The planes of $T_{2}-T_{2}$ exchange data are then transformed using a two-dimensional (2D) inverse Laplace algorithm.

\section{B. Inverse Laplace transform}

We use the inverse Laplace transform to obtain the distribution of $T_{2}$ times in the sample. The inverse Laplace transform [12] may be compared to the well known Fourier transform. Whereas the Fourier transform provides the frequency components of an oscillating signal, the inverse Laplace transform gives the characteristic times of a decaying exponential. A vital difference is that the Fourier transform is well posed while the inverse Laplace is not, there being only one possible solution for a Fourier transform while there are many possible fits of characteristic times for a decaying exponential. Regularization is needed to ensure a robust solution to the inverse problem in addition to preventing noise from adversely affecting the results. For all our experiments, we use the Tikhonov regularization method [13].

The development of the 2D inverse Laplace transform [14] has led to the advent of a wide range of separation [15], correlation [16], and exchange NMR experiments [17]. The 2D inverse Laplace transform is a Fredholm integral of the first kind, given by the structure

$$
M\left(\tau_{1}, \tau_{2}\right)=\iint k_{1}\left(\tau_{1}, x\right) k_{2}\left(\tau_{2}, y\right) F(x, y) d x d y+\epsilon\left(\tau_{1}, \tau_{2}\right),
$$

where $k_{1}$ and $k_{2}$ are the separable kernels, $F$ is the probability density, $x$ and $y$ are the chosen domain for solving $F(x, y)$, and $\epsilon$ is the experimental noise. For the case of our transverse relaxation exchange experiment, the equation describing the magnetization is given by

$$
\begin{aligned}
M\left(\tau_{n}, \tau_{m}\right)= & \iint k_{1}\left(\tau_{n}, T_{2}^{1}\right) k_{2}\left(\tau_{n}, T_{2}^{2}\right) F\left(T_{2}^{1}, T_{2}^{2}\right) d T_{2}^{1} d T_{2}^{2} \\
& +\epsilon\left(\tau_{n}, \tau_{m}\right),
\end{aligned}
$$

where $T_{2}^{1}$ and $T_{2}^{2}$ are the measured $T_{2}$ values from the first and second encoding intervals, respectively. The transformed 2D inverse Laplace exchange spectra are similar in form to their Fourier exchange cousins, such as the NOESY or
ROESY experiments. Peaks that appear along the diagonal correspond to spin-bearing molecules that are still in their original environment during the second encode interval. Offdiagonal peaks are from the spins which have shifted environments during the mixing time.

A major point of deliberation for our experiments concerned consistency of regularization. The non-negative leastsquares algorithm (NNLS) used in the inverse Laplace transform tends to return solutions consisting of numerous sharp peaks. A regularization parameter is used to force the algorithm to return solutions that have a smooth spectrum. The parameter $\alpha$ regulates the amount of smoothing applied to the spectrum. In most situations, the value of $\alpha$ would be adjusted to just minimize $\chi^{2}$ (and no further) for each spectrum. However, as we are tracking integrated peak intensities over numerous spectra, a single common value of $\alpha$ is chosen for consistency. The use of a common $\alpha$ value for all the spectra is not expected to have a significant impact in the results, as the difference in individually optimized $\alpha$ values was slight.

One behavior of note is the tendency of the inverse Laplace transform to turn an expected continuous distribution into several discrete spectral peaks, an effect known as "pearling." This effect is an artifact. The tendency of the inverse Laplace transform to break up a continuous distribution into discrete peaks makes it a useful artifact where we wish to represent the pore size in a discrete manner.

\section{X-ray computed tomography}

An alternative means of probing the morphology of the pore space is by direct imaging. Here we use micro x-ray computed tomography and subsequent analysis to provide validation for our NMR results. The process is illustrated in Fig. 2 using the sample of this study. An x-ray density map is acquired [Fig. 2(a)], segmented into phases [Fig. 2(b)], followed by the calculation of the covering radius map [Fig. 2(c)] and a partitioning of the pore space into simple geometric cells [Fig. 2(d)] [18]. For an extended description of the facility and a recent review see $[19,20]$.

We compare in Fig. 3 the distribution function of the covering radius map, an approach of estimating pore size without requiring explicit pore partitioning, with the pore size distribution based on pore partitioning. Both pore size distributions (PSDs) exhibit a similar mean. The PSD based on covering radius is slightly broader and shows higher occupation at lower radii. This is indicative of pores not being entirely spherical entities, e.g., they can exhibit roughness and are not isolated, the connections between pores "throats" generally being more narrow.

In Fig. 4 we use the explicit partitioning of the pore space into pores [18] to derive the distances of the centers of neighboring pores. As the center of the pore we take either the center of mass (noted "cmas"), or the center of the largest sphere which can fit into the pore by use of the Euclidean distance transform (noted "edt"). The pore-pore distances are volume weighted by the average of the pore volumes associated with the respective pore-pore links. Figure 4 also shows the distributions of pore-pore distances projected onto 

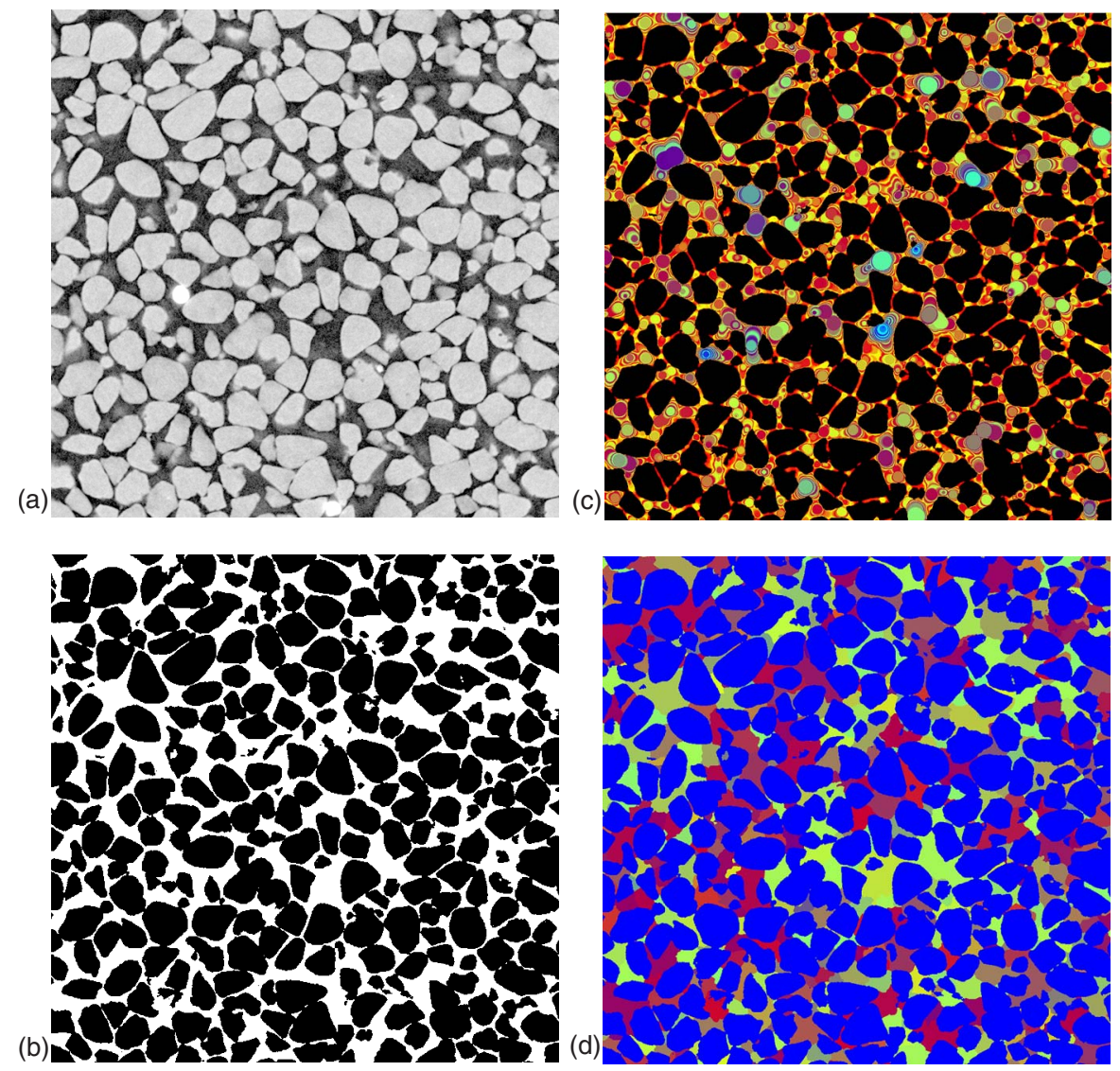

FIG. 2. (Color online) X-ray CT central slice of the tightpacked quartz sand $(3.4 \mathrm{~mm}$ by $3.4 \mathrm{~mm}$ or $1000 \times 1000$ voxel). (a) X-ray density map. (b) Segmentation into grain and void space. [(c) and (d)] Covering radius map and pore partition of the void space. the tomogram axes, the maximum of those distributions naturally being closer to the origin.

To account for the tortuosity of the pathways between pores we solve the Laplace equation for diffusivity of the whole structure directly on the image using a conjugate gradient method [21]. Figure 5 depicts the tortuosity $T_{\sigma}=\phi \frac{\sigma_{0}}{\sigma_{\text {eff }}}$, where $\sigma_{0}$ is the conductivity of the fluid, $\sigma_{\text {eff }}$ is the effective conductivity of the subdomain, and $\phi$ porosity. We present results for calculations at different scales and remark, that for smaller scales, as relevant for NMR diffusion measurements, a larger spread of tortuosity values could be expected. The average tortuosity at the full image scale $(3.4 \mathrm{~mm})$ is about 1.7. Noting that tortuosity is only a mild function of porosity, a reasonable estimate of the length of the tortuous pathway between pores is given by the product of pore distance and tortuosity.

\section{ANALYSIS}

The $T_{2}-T_{2}$ exchange spectra show remarkable differences depending on mixing time and displacement. Figure 6 shows the exchange plots for various mixing times and displacements. For short mixing times, the signal mainly lies along the diagonal, indicating that the spin-bearing fluid molecules are still in their original environments. A slight skewing off the diagonal can be seen for the shortest $T_{2}$ peak for short mixing times and small displacement. We are unsure as to the cause of this skewing effect, presented in a subsequent paper. The $T_{2}-T_{2}$ exchange plots at these short mixing peri- ods start to show evidence for pore-to-pore exchange for displacements of about 25-30 $\mu \mathrm{m}$. As we move to longer mixing times, we see that the off-diagonal intensity increases, indicating that more fluid molecules have managed to shift $T_{2}$ environments. We also start to see off-diagonal intensity arising at smaller displacements. By $\tau_{m}=250 \mathrm{~ms}$, we begin to see off-diagonal peaks form even at the smallest displacements. This at first seems contradictory, as molecules that have not moved should still be in their original $T_{2}$ environment. However, it is important to note that the porous medium is isotropic and we are encoding for movement in one direction only. Therefore, it is possible for molecules to move to a new environment perpendicular to the diffusion axis while having traveled very little distance along it.

An interesting effect is that the peaks become more discretized for both large displacements and long mixing times, changing from a continuous signal along the diagonal to three individual peaks. We speculate that this could be due to a $T_{1}$ averaging effect. For short times and displacements, the fluid molecules have only experienced a small portion of the pore surface. At longer displacements and times, molecules are likely to have experienced more of the pore surface, leading to a better averaged $T_{1}$ time, thus helping to discretize the peaks in the $T_{2}$ exchange spectrum.

We now turn to quantitative analysis of our NMR data. For convenience we focus our attention on a subset of the pore distribution created by the ILT pearling effect, namely, the two classes of pores labeled $\alpha$ and $\beta$, as indicated in Fig. 6(h). We integrate the different peak intensities for the different mixing times and displacements, combining intensities 

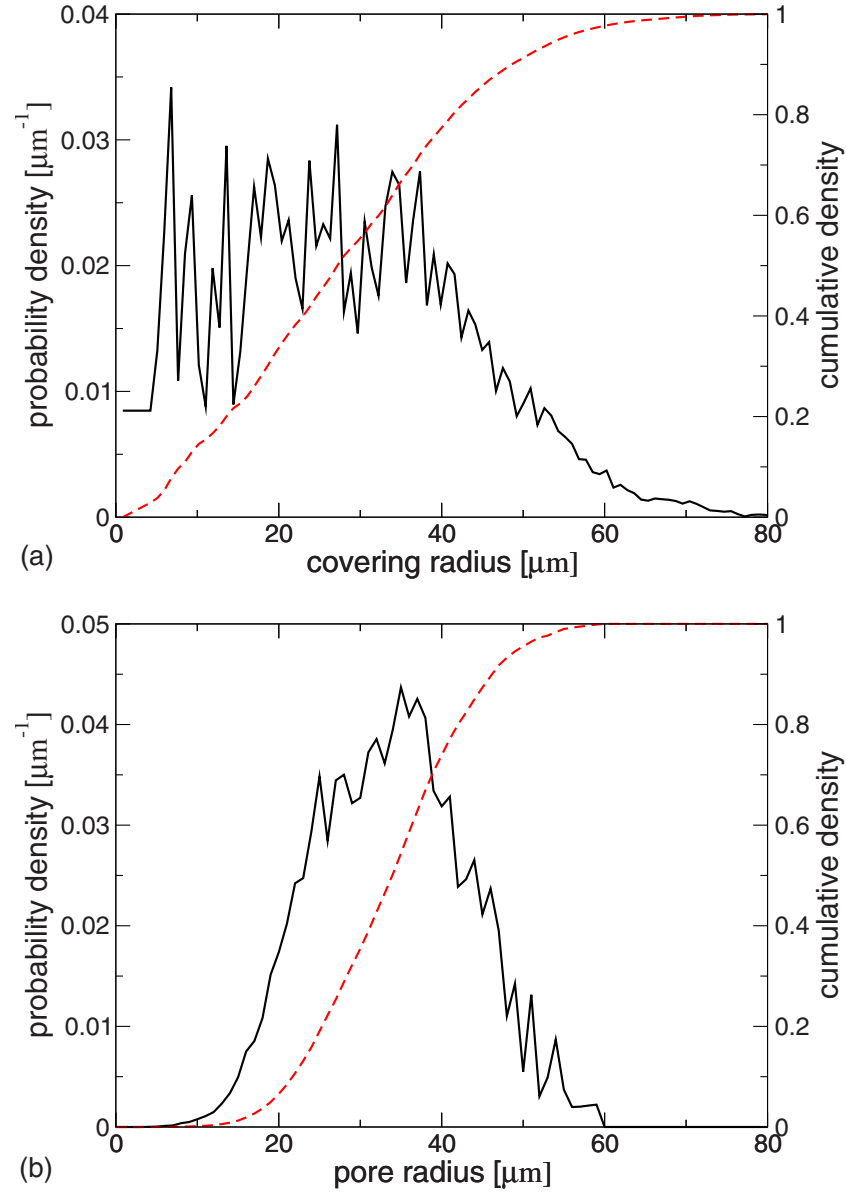

FIG. 3. (Color online) Pore size distribution for tight-packed quartz sand found through (a) the covering radius and (b) the pore partitioning method. Straight lines note the probability density functions.

for the complementary (symmetric) exchange peaks. We then normalize the peak intensities as a function of the total integrated value at that mixing time so as to remove influence of $T_{1}$ relaxation. These normalized intensities are then plotted

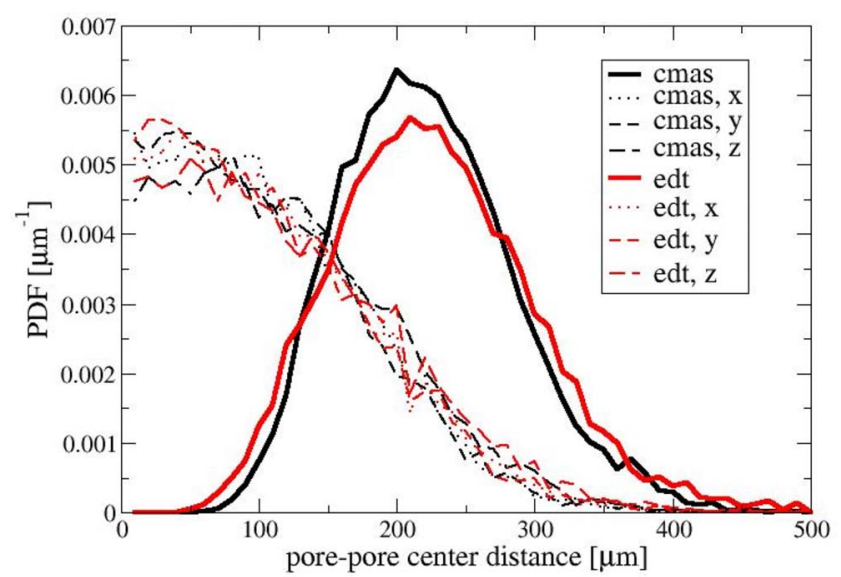

FIG. 4. (Color online) Interpore spacing for tight-packed quartz sand. Solid lines are absolute distances while dotted lines are projected along a single axis. cmas refers to center-of-mass calculations and edt is from Euclidean distance transformation.

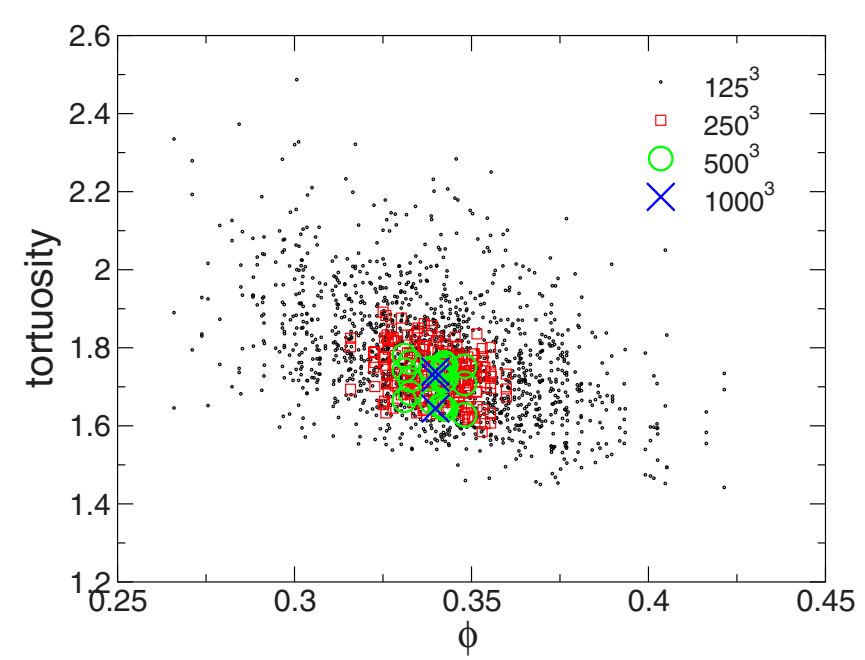

FIG. 5. (Color online) Tortuosity as function of total porosity $\phi$ and domain size (given in the legend in units of voxel cubed) based on the solution of the Laplace equation for electrical conductivity or diffusivity.

as a function of mixing time and displacement as seen in Fig. 7. These intensities effectively represent probability distributions or propagators, $P^{\alpha \beta}$, that a molecule starting in pore $\alpha$ and ending in pore $\beta$ has displaced a distance $Z$ over the experimental mixing time.

\section{THEORY}

We idealize the pore system in terms of rapid diffusion within discrete classes of pores labeled by local surface to volume ratio, and hence $T_{2}$ value, using an exponent $\alpha, \beta$ to label the class of pore size. The diffusion between different pores over the mixing time $\tau_{m}$ is assumed to occur at a much slower rate. The fast intrapore diffusion (pore equilibration) assumption will require pores to have dimensions on the order of or smaller than $\sqrt{D_{0} \tau_{m}}$, where $D_{0}$ is the free molecular self-diffusion coefficient. This condition is satisfied only for pores smaller than $20 \mu \mathrm{m}$ at the mixing times used here. We discuss later how we deal with breakdown of the pore equilibrium assumption. The advantage of assuming pore equilibration is that the echo attenuation may be reduced to a simple product of a pore structure factor [6], $\left|S_{0}^{\alpha}(q)\right|^{2}$, and the Fourier transform of the pore-to-pore propagator, $P_{p p}\left(Z, \tau_{m}\right)$, representing displacements between pores. Obviously, $\left|S_{0}^{\alpha}(q)\right|^{2}$ will depend on the pore size, while $P_{p p}\left(Z, \tau_{m}\right)$ will depend on the occupancies of the various pores to which molecules migrate and the relative displacements of those pores to the respective origin pores of the migrating molecules. Different values of $P_{p p}\left(Z, \tau_{m}\right)$ apply depending on whether the exchange is between pores of similar size or differing size.

\section{A. Exchange between similar size pores}

We start by considering hopping between pores of the same class, and which therefore have the same $T_{2}$ value, relating to intensity along the diagonal of the $T_{2}-T_{2}$ plane. 

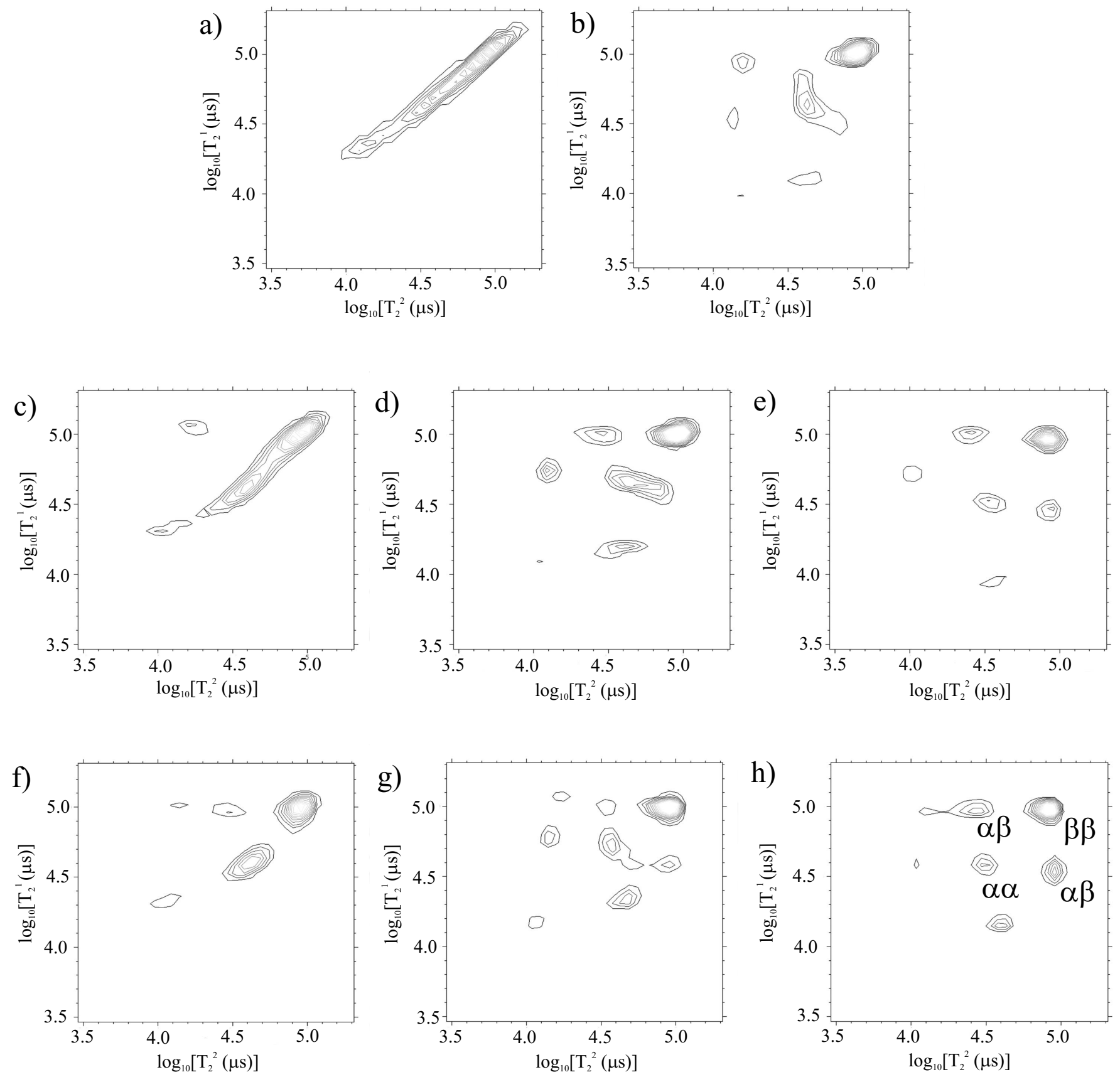

FIG. 6. Exchange spectra (a) $100 \mathrm{~ms}$ exchange time, $0 \mu \mathrm{m}$ displacement, (b) $100 \mathrm{~ms}$ exchange time, $30 \mu \mathrm{m}$ displacement, (c) $250 \mathrm{~ms}$ exchange time, $0 \mu \mathrm{m}$ displacement, (d) $250 \mathrm{~ms}$ exchange time, $30 \mu \mathrm{m}$ displacement, (e) $250 \mathrm{~ms}$ exchange time, $45 \mu \mathrm{m}$ displacement, (f) $500 \mathrm{~ms}$ exchange time, $0 \mu \mathrm{m}$ displacement, (g) $500 \mathrm{~ms}$ exchange time, $30 \mu \mathrm{m}$ displacement, and (h) $500 \mathrm{~ms}$ exchange time, $45 \mu \mathrm{m}$ displacement. Note, there is no exchange plot at $45 \mu \mathrm{m}$ for the $100 \mathrm{~ms}$ exchange time, as fluid molecules will not have diffused that far for that mixing period.

We shall further assume an orientationally disordered pore glass, for which displacements have a mean length $b$ but no orientational order [6]. The diffusion echo attenuation is described by

$$
M^{\alpha \alpha}(q, \Delta)=\left|S_{0}^{\alpha}(q)\right|^{2} \mathfrak{I}\left(C_{0}^{\alpha}+\sum_{n=1}^{\infty} C_{n}^{\alpha \alpha} H\left(Z, n b_{\alpha \alpha}\right)\right),
$$

where $C_{n}$ is the probability of hopping to the $n$th neighbor shell, $\mathfrak{I}$ is the Fourier transform, $b$ is the pore spacing, and $H$ is the probability distribution of pore centers for the $n$th pore shell along $Z$. For an isotropic medium as used in this work, this distribution will be uniform across a range $-n b<Z<$ $+n b$ and can be modeled as a Heaviside hat function. Because the signal from molecules which remain in their original pores and the signal from molecules that have exchanged with pores of the same class both lie upon the diagonal in the $T_{2}-T_{2}$ plane, we will have a nonzero starting intensity. Note that Eq. (9) generates the case of $N=0$ for which no migration has occurred, allowing for the separation of 

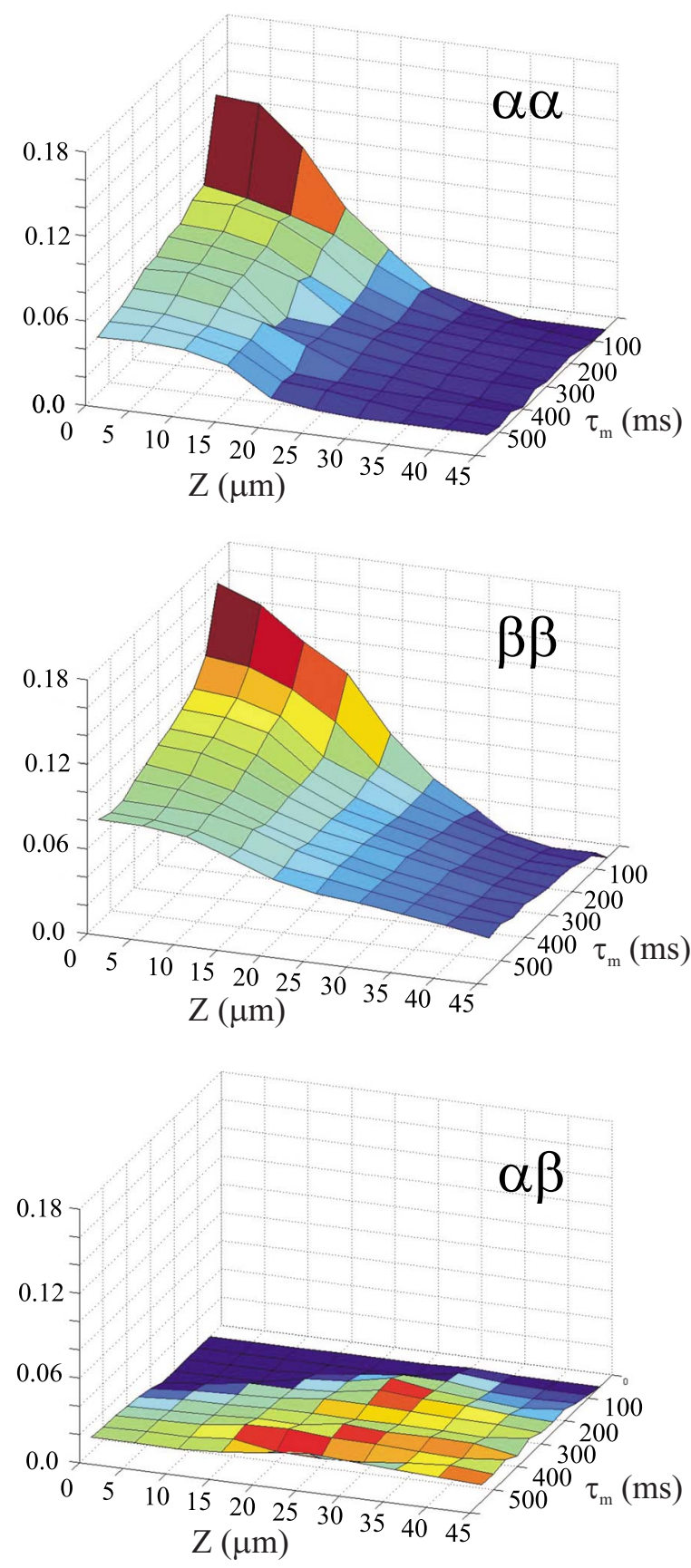

FIG. 7. (Color online) Normalized experimental intensity plots for propagators $P^{\alpha \alpha}, P^{\beta \beta}$, and $P^{\alpha \beta}$, describing the probability that spin-bearing molecules migrate a distance $Z$ along the magnetic field gradient direction in a time $\tau_{m}$ corresponding to the experimental mixing time.

the contribution to the magnetization from the molecules in their original pores from the signal of molecules that have shifted environments. Evaluation of the Fourier transform in Eq. (9) gives

$$
M^{\alpha \alpha}(q, \Delta)=\left|S_{0}^{\alpha}(q)\right|^{2}\left[C_{0}^{\alpha}+\sum_{n} C_{n}^{\alpha \alpha} \frac{\sin \left(2 \pi q n b_{\alpha \alpha}\right)}{2 \pi q n b_{\alpha \alpha}}\right] .
$$

The one-dimensional form factor $\left|S_{0}^{\alpha}(q)\right|^{2}$ is the squared Fourier transform of the density function, which makes it depen- dent upon both pore size and pore shape. We assume for simplicity spherical pores. Note that such a shape cannot possibly be accurate in real porous materials. However, the assumption of sphericity is consistent with pore isotropy, the lack of any preferred axis in the medium. At best, such a pore structure factor provides a means of assigning an isotropic pore length scale. The density function of a sphere projected along a one-dimensional axis of radius $r$ is described by

$$
\rho(z)=\left(\frac{3}{r^{3}}\right)\left(r^{2}-z^{2}\right)
$$

for $-r<z<r$ The measured signal in an experiment would be the Fourier transform of this distribution, given by

$$
\begin{aligned}
\left|S_{0}^{\alpha}(q)\right|^{2} & =\int_{-r}^{2}\left(\frac{3}{4 r^{3}}\right)\left(r^{2}-z^{2}\right) \exp (2 \pi i q z) d z \\
& =\frac{3[\sin (2 \pi q r)-(2 \pi q r) \cos (2 \pi q r)]}{(2 \pi q r)^{3}} .
\end{aligned}
$$

Our form factor is $\left|S_{0}^{\alpha}(q)\right|^{2}$ and has a node when $q r \approx \frac{3}{4}$. When $q \ll r^{-1}$, the form factor can be approximated by

$$
\left|S_{0}^{\alpha}(q)\right|^{2}=1-\frac{1}{5}(2 \pi q r)^{2},
$$

which then simplifies to

$$
\left|S_{0}^{\alpha}(q)\right|^{2}=\exp \left(-\frac{1}{5}(2 \pi q)^{2} r^{2}\right)
$$

We rewrite this equation in terms of $\sigma^{2}=\frac{2}{5} r^{2}$ to obtain

$$
\left|S_{0}^{\alpha}(q)\right|^{2}=\exp \left(-\frac{1}{2}(2 \pi q)^{2} \sigma_{\alpha}^{2}\right) .
$$

This form of the equation will simplify later Fourier transform calculations. We then substitute Eq. (15) into Eq. (10) to obtain the equation

$$
\begin{aligned}
M^{\alpha \alpha}(q, \Delta)= & \exp \left(-\frac{1}{2}(2 \pi q)^{2} \sigma_{\alpha}^{2}\right) \\
& \times\left[C_{0}^{\alpha}+\sum_{n} C_{n}^{\alpha \alpha} \frac{\sin \left(2 \pi q n b_{\alpha \alpha}\right)}{2 \pi q n b_{\alpha \alpha}}\right] .
\end{aligned}
$$

We take the Fourier transform of Eq. (16) with respect to $q$ to obtain the overall propagator as follows:

$$
\begin{aligned}
P^{\alpha \alpha}(Z, \Delta)= & C_{0}^{\alpha} \rho_{0}^{\alpha}(Z) * \rho_{0}^{\alpha}(Z)+\sum_{n} C_{n}^{\alpha \alpha}\left[\rho_{0}^{\alpha}(Z) * \rho_{0}^{\alpha}(Z)\right] \\
& \otimes H\left(Z, n b_{\alpha \alpha}\right)
\end{aligned}
$$

where $*$ denotes a spatial correlation function and $\otimes$ denotes a spatial convolution. $P^{\alpha \alpha}$ gives us the distribution of molecular movement as determined by the restrictions in the sample due to pore configuration. For molecules remaining in their original pores, this is simply the pore occupancy multiplied by the pore correlation function. For molecules that have moved to a new pore, the distribution of pores also plays a part. We now need to solve for this convolution of the 
density function with the hat function in terms of interpore spacing and pore size

$$
\begin{aligned}
& {\left[\rho_{0}^{\alpha}(Z) * \rho_{0}^{\alpha}(Z)\right] \otimes H\left(Z, n b_{\alpha \alpha}\right)} \\
& \quad=\frac{1}{4 n b_{\alpha \alpha}}\left[\operatorname{erf}\left[\frac{Z+n b_{\alpha \alpha}}{\sqrt{2} \sigma_{\alpha}}\right] \pm \operatorname{erf}\left[\frac{Z-n b_{\alpha \alpha}}{\sqrt{2} \sigma_{\alpha}}\right]\right],
\end{aligned}
$$

where $\rho^{\alpha}$ is the local density of the pore $\alpha$. Equation (18) simplifies to one of two equations depending whether $Z$ is larger or smaller than the pore spacing, with a positive sign for the case of $Z<n b$ and a negative sign for $Z>n b$. Note that Eq. (15) only holds true in the fast diffusion limit, where all the molecules have diffused the distance length of the intrapore dimension. At our shortest mixing times, the pores in our sample are large enough that we are not in the fast diffusion limit. For these cases, one method to deal with this situation is to multiply the pore density function by the diffusive envelope

$$
\left(4 \pi D_{0} \Delta\right)^{-1 / 2} \exp \left[\frac{-Z^{2}}{4 D_{0} \Delta}\right]
$$

We multiply Eq. (18) by this function. We take as $D_{0}$ the diffusion coefficient of water at room temperature, which is $2.3 \times 10^{-9} \mathrm{~m}^{2} \mathrm{~s}^{-1}$ in the present context. This becomes a constant multiplier once the fast diffusion limit is reached.

\section{B. Exchange between different size pores}

We now turn to the off-diagonal peaks in the $T_{2}-T_{2}$ plane. For migration between pores of different $T_{2}$ values, the behavior is similar to that in Eq. (9) but without the term for molecule residency in the starting pore. For this case, the echo attenuation can simply be described by

$$
M^{\alpha \beta}(q, \Delta)=\left|S_{0}^{\alpha}(q)\right|\left|S_{0}^{\beta}(q)\right|\left[\sum_{n} C_{n}^{\alpha \beta} \frac{\sin \left(2 \pi q n b_{\alpha \beta}\right)}{2 \pi q n b_{\alpha \beta}}\right] .
$$

Between pores of differing sizes, the form factor is a combination of both pore sizes. Again, we assume the case of spherical pores as follows:

$$
S_{0}^{\alpha}(q) S_{0}^{\beta}(q)^{*} \approx \exp \left(-\frac{1}{5}(2 \pi q)^{2} \frac{1}{2}\left(r_{\alpha}^{2}+r_{\beta}^{2}\right)\right) .
$$

To simplify the Fourier transform, we rewrite Eq. (21) as a function of $\sigma_{\alpha \beta}^{2}=\frac{1}{5}\left(r_{\alpha}^{2}+r_{\beta}^{2}\right)$. The echo attenuation can be rewritten as

$$
M^{\alpha \beta}(q, \Delta)=\exp \left(-\frac{1}{2}(2 \pi q)^{2} \sigma_{\alpha \beta}^{2}\right)\left[\sum_{n} C_{n}^{\alpha \beta} \frac{\sin \left(2 \pi q n b_{\alpha \beta}\right)}{2 \pi q n b}\right] .
$$

We Fourier transform Eq. (22) to obtain the average propagator. We use a positive sign for $Z<n b$ and a negative sign for $Z>n b$.

$$
\begin{aligned}
P^{\alpha \beta}(Z, \Delta)= & \sum_{n} C_{n}^{\alpha \beta} \frac{1}{4 n b_{\alpha \beta}}\left[\operatorname{erf}\left[\frac{Z+n b_{\alpha \beta}}{\sqrt{2} \sigma_{\alpha \beta}}\right]\right. \\
& \left. \pm \operatorname{erf}\left[\frac{Z-n b_{\alpha \beta}}{\sqrt{2} \sigma_{\alpha \beta}}\right]\right] .
\end{aligned}
$$

Once again, we need to multiply the result by the diffusive envelope of Eq. (19) to account for the fact we are not in the fast diffusion limit.

\section{SIMULATIONS OF INTERPORE DIFFUSION}

To interpret our data, we require a representation of the pore-hopping probability $C_{n}$ to mimic the movement of fluid between pores. Two models were developed: an analytic model, which uses spreading Gaussians to determine pore occupancy and a probabilistic hopping model, where molecules have a finite chance of moving to a new pore at each time step. Both sets of simulations were carried out in MATLAB, the computed probability first roughly fitted to the experimental data by hand, and then optimized using the Nelder-Mead method [22]. This technique serves to minimize a function of several variables, which in our case is a chi squared relation

$$
\chi^{2}=\left(P_{\exp }-P_{\text {sim }}\right)^{2}
$$

where $\chi$ is the squared difference between the experimental and simulated results. For our model, the variables were the different pore sizes $\left(r_{\alpha}, r_{\beta}\right)$, pore spacings $\left(b_{\alpha \alpha}, b_{\alpha \beta}, b_{\beta \beta}\right)$, and interpore diffusion coefficients $\left(D_{\alpha \alpha}, D_{\alpha \beta}, D_{\beta \beta}\right)$. To find the error bars, we found the deviation from the optimized value needed to double $\chi^{2}$. The probabilistic nature of the pore-hopping simulations causes minor variations in chi squared, even for identical starting parameters, such that calculation of precise error bars was not possible.

For our sand, we assume a two-pore system reflecting the small number of peaks in the $T_{2}-T_{2}$ spectra. We justify this assumption by noting that the signal intensity from the shortest $T_{2}$ peak comprises only a small percentage of total signal intensity. We refer to the smaller pores as $\alpha$ pores and the larger pores as $\beta$ pores. In an isotropic distribution around our starting $\alpha$ and $\beta$ pores, there exist corresponding $\alpha$ and $\beta$ pores. We refer to this first shell of pores as $\alpha^{\prime}$ or $\beta^{\prime}$ pores. Around these pores there is yet another shell, $\alpha^{\prime \prime}$ and $\beta^{\prime \prime}$. We only consider the shells as far out as the second nearest neighbor since movement beyond the second pore is extremely unlikely at the time scales with which we are working.

\section{A. Analytic simulation}

A simplistic approach to representing the interpore occupancies $C_{n}$ is to use a simple diffusive envelope, where the relevant diffusion coefficient is the time for interpore migration. For the case of movement between pores of the same class, this probability is represented as a spreading Gaussian 


$$
C_{n}^{\alpha \alpha}=\frac{4 \pi\left(n b_{\alpha \alpha}\right)^{2} b_{\alpha h a}}{\left(4 \pi D_{p}^{\alpha \alpha} \Delta\right)^{3 / 2}} \exp \left(-\frac{n^{2} b_{\alpha \alpha}^{2}}{4 D_{p}^{\alpha \alpha} \Delta}\right),
$$

where $n$ is the pore shell, $b$ is the mean pore spacing, and $D_{p}$ is the interpore diffusion coefficient. The equation for the case of exchange between pores of differing sizes is similar, except it now uses the mean pore spacing and interpore diffusion coefficient between pores of different classes

$$
C_{n}^{\alpha \beta}=\frac{4 \pi\left(n b_{\alpha \beta}\right)^{2} b_{\alpha \beta}}{\left(4 \pi D_{p}^{\alpha \beta} \Delta\right)^{3 / 2}} \exp \left(-\frac{n^{2} b_{\alpha \beta}^{2}}{4 D_{p}^{\alpha \beta} \Delta}\right) .
$$

Unlike the other two occupancies, which will grow with time, the occupancy of the origin pores will decrease. To calculate the residency in the original pore, we subtract the two interpore occupancies

$$
C_{0}^{\alpha} \approx 1-\sum_{n=1} C_{n}^{\alpha \alpha}-\sum_{n=1} C_{n}^{\alpha \beta}
$$

\section{B. Probabilistic simulation}

For our probabilistic simulations, we fill the pores and pore shells with molecular walkers. At the end of the simulation, though, we will only consider the movement of molecules starting from $\alpha$ or $\beta$ pores, which we consider origin pores. For each time step in the simulation, a molecule has a certain likelihood that it will jump from the pore it is in to a neighboring pore. The balance of mass must be maintained as our system is fully saturated. To do this, a molecule in the new pore must exchange with the hopping molecule. The program randomly searches for a molecule in the new pore to place into the hopping molecule's original pore. At the end of the experiment, we tally the locations of molecules from the original $\alpha$ and $\beta$ pores for each time step. The number of molecules in each location is then substituted for $C_{n}$ in the propagator equation in our pore-hopping simulation. We can then apply these occupancies in a simulation which solves the average propagator equation to find the fluid movement within the sample over time.

\section{RESULTS}

On suitable variation of the fitting parameters, the simulated results, shown in Figs. 8 and 9, show good agreement with the experimental data in Fig. 7, the poorest result being for exchange between pores of differing size (off-diagonal peaks) at short mixing times. We believe that this discrepancy at short mixing times between the simulated and experimental data for exchange between pores of differing size arises from resolution problems in the inverse Laplace transform where the off-diagonal peak is of weak intensity. In the presence of a peak of much greater intensity, the inverse Laplace transform often cannot resolve a weak intensity peak. Therefore, until the weaker peak has reached a sufficient intensity compared to the greater peak, the transform cannot resolve it. As our simulations require matching of intensities, this discrepancy has led to some uncertainty in some of the values of the interpore spacing and interpore diffusion coefficient between $\alpha$ and $\beta$ pores.
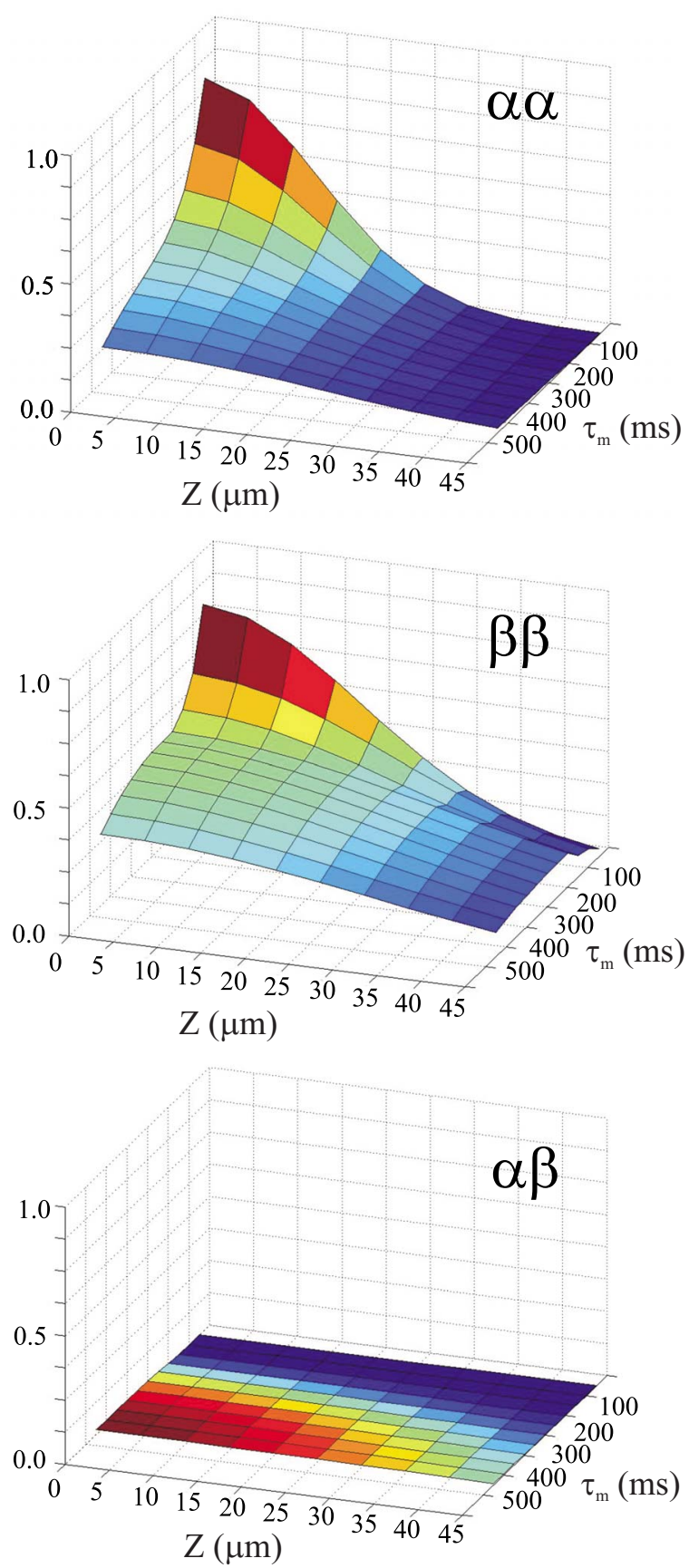

FIG. 8. (Color online) Analytic simulation intensity plots.

\section{A. Diagram interpretation}

The fitting process was based on a least-squares minimization right across the $\left(Z, \tau_{m}\right)$ plane of peak intensity. However, specific parameters of the fit influence different features of the distributions, thus simplifying the search for suitable initial parameters in the multidimensional parameter space, and thus assisting in the search for a global minimum. Figure 10 gives an annotated peak intensity diagram that shows how to interpret the plots. The vertical axis is the normalized peak intensity. The displacement axis $(Z)$ shows how the intensity of a peak evolves depending on distance. The mixing time axis $\left(\tau_{m}\right)$ shows how the peak intensity behaves as a function 

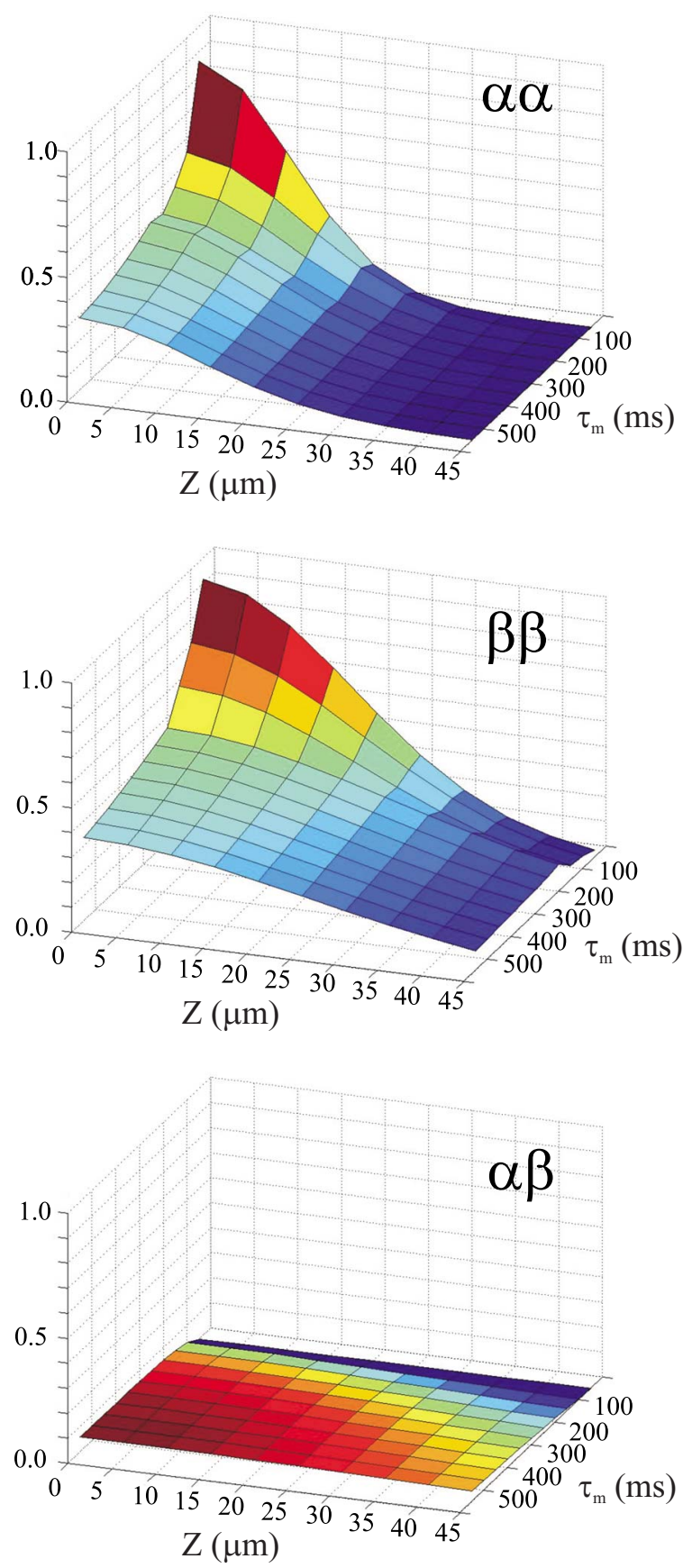

FIG. 9. (Color online) Probabilistic simulation intensity plots.

of time. At small displacements, the decrease in signal intensity, or increase for exchange between unlike pores, is governed by interpore diffusion. For short mixing times, the signal intensity along the displacement axis is influenced mostly by pore radius. For longer mixing times and finite displacements, the spread of intensity is governed by the interpore spacing.

\section{B. Pore radii}

Provided the amount of exchange is small, pore radii for pores smaller than the diffusion length scale of the experi-

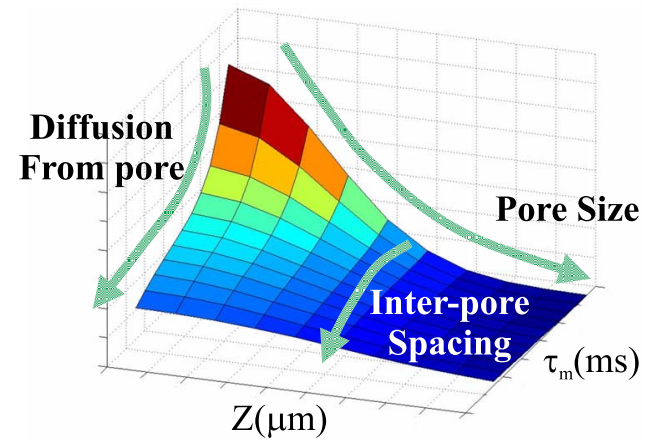

FIG. 10. (Color online) Annotated peak intensity diagram. The $\tau_{m}$ axis is increasing mixing time, the $P$ axis is increasing displacement, and the $Z$ axis is the normalized intensity.

ment can almost be read directly from the experimental results at small values of $\tau_{m}$. For short mixing periods, the intensity will temporarily stop spreading along the displacement axis once $\tau_{m}$ is sufficiently large that the molecules have collided with the starting pore surface, which is displaced on the order of the pore radius. From our probabilistic simulations, we estimate that the pore radius size for the smaller $\alpha T_{2}$ peak is on the order of $24 \mu \mathrm{m}$. The analytic simulations agree with this value, also producing a pore radius size of $25 \mu \mathrm{m}$ (see Table I). For pore radii larger than the diffusion length scale of the experiment, such simple manifestation of the pore size is not apparent at $\tau_{m}=100$. Here we must analyze our distributions across the $\left(Z, \tau_{m}\right)$ plane by looking at how pore size affects the spread of intensity. The more quickly the intensity continues to spread, the larger the pore size. By matching our simulated intensity spreads we were able to estimate our $\beta$ pore sizes. The probabilistic and analytic simulations gave $\beta$ pore sizes of around $49 \mu \mathrm{m}$ and $52 \mu \mathrm{m}$, respectively. These calculated values of $\alpha$ and $\beta$ are consistent with the pore size distribution obtained from the $\mathrm{x}$-ray computed tomography (CT) data.

\section{Interpore spacing}

The effects of interpore spacing are subtle in the case of exchange between like pores, except in the situation of very fast diffusion. The interpore spacing strongly influences the intensity at long mixing times, where signal has begun to spread beyond the distance of the estimated pore space. The further the intensity distribution along $Z$ spreads along the diffusion axis, the larger the interpore distance. The interpore spacing also has an influence on the rate of intensity decay. If

TABLE I. Pore properties.

\begin{tabular}{lcc}
\hline \hline \multicolumn{1}{c}{$T_{2}$} & $\alpha(\mu \mathrm{m})$ & $\beta(\mu \mathrm{m})$ \\
\hline & 38 & 79 \\
Pore radius & $r_{\alpha}(\mu \mathrm{m})$ & $r_{\beta}(\mu \mathrm{m})$ \\
Analytic & $25 \pm 3$ & $52 \pm 13$ \\
Probabilistic & 24 & 49 \\
\hline \hline
\end{tabular}


TABLE II. Interpore properties.

\begin{tabular}{lccc}
\hline \hline Interpore spacing & $b_{\alpha \alpha}(\mu \mathrm{m})$ & $b_{\beta \beta}(\mu \mathrm{m})$ & $b_{\alpha \beta}(\mu \mathrm{m})(\mathrm{s})$ \\
\hline Analytic & $51 \pm 9$ & $134 \pm 37$ & $83 \pm 15$ \\
Probabilistic & 56 & 108 & 76 \\
Characteristic times & $\lambda_{\alpha \alpha}(\mathrm{s})$ & $\lambda_{\beta \beta}(\mathrm{s})$ & $\lambda_{\alpha \beta}(\mathrm{s})$ \\
Analytic & 1.35 & 3.2 & 0.63 \\
Probabilistic & 2.63 & 2.38 & 0.71 \\
$D_{p}$ & $D_{\alpha \alpha}\left(\frac{m^{2}}{s}\right)$ & $D_{\beta \beta}\left(\frac{m^{2}}{s}\right)$ & $D_{\alpha \beta}\left(\frac{m^{2}}{s}\right)$ \\
Analytic & $3.2 \times 10^{-10}$ & $9.3 \times 10^{-10}$ & $18.0 \times 10^{-10}$ \\
Probabilistic & $1.9 \times 10^{-10}$ & $10.8 \times 10^{-10}$ & $13.6 \times 10^{-10}$ \\
Tortuosity & $T_{\alpha \alpha}$ & $T_{\beta \beta}$ & $T_{\alpha \beta}$ \\
Analytic & 7.1 & 2.5 & 1.3 \\
Probabilistic & 11.5 & 2.1 & 1.8 \\
\hline \hline
\end{tabular}

the pores are very far apart, the likelihood of moving from one pore to another is less, so the signal decays more slowly. Clearly the interplay between decay along the $\tau_{m}$ and $Z$ axes is sensitive to both interpore spacing and interpore diffusion rate. Note, that it is, in principle, possible to estimate pore spacings greater than the molecular diffusion distance because of this interplay, though accuracy may be impeded.

Finding the interpore spacing between unlike pores is similar to finding the pore radius. For pore spacings less than the diffusion distance of the experiment, the signal will stop advancing at approximately the interpore spacing. For pore spacing larger than the diffusion resolution, we look at how the signal advances. The distance between the $\alpha$ and $\beta$ pores in our system appears to be beyond our measured diffusion lengths.

The pore-hopping model fit found a distance of $b_{\alpha \beta}$ $=76 \mu \mathrm{m}$ while the analytic model had a larger spacing of $b_{\alpha \beta}=83 \mu \mathrm{m}$ (Table II). Unfortunately, due to previously discussed resolution issues of the inverse Laplace, we need to be cautious in regards to the accuracy of the average interpore spacing between $\alpha$ and $\beta$ pores.

The optimization for exchange between pores of a similar class returned interesting behavior. Above certain interpore spacings, $\chi^{2}$ would reach a minimum, but in a broad valley where the value of $\chi^{2}$ was constant extending to large values of $b_{\alpha \alpha}$ and $b_{\beta \beta}$. We believe this behavior can give an estimate of the possible minimum interpore spacing, but we are unsure as to the upper limits of the fit. We estimate the minimum interpore spacing between $\alpha$ pores to be $b_{\alpha \alpha}=56 \mu \mathrm{m}$ from our pore-hopping model and $b_{\alpha \alpha}=51 \mu \mathrm{m}$ from our analytic model (Table II). The probabilistic model returned a minimum value of $b_{\beta \beta}=108 \mu \mathrm{m}$ and the analytic model $b_{\beta \beta}=134 \mu \mathrm{m}$. These minimum values are later used for calculations of other pore characteristics.

While the interpore spacings we calculate are consistent with the interpore spacings seen in the $\mathrm{x}$-ray $\mathrm{CT}$ data, there exist many pores with connection distances larger than those found with our data. Because we are limited by the diffusion distance of the molecules, the nearest neighbor pores at the longer distances may not be adequately probed. We believe the wide valleys in our simulated fits reflect our inability to investigate these length scales. We anticipate that driven fluid flow through the sand will increase the number of molecules exchanging pores, allowing the exchange peaks to be better resolved by the inverse Laplace and giving them stronger intensity in the exchange plots, and thus allow us to more accurately determine interpore spacing.

\section{Rate of exchange between pores}

The rate at which intensity decays along the $Z$ axis for the pore-hopping simulations reflects how quickly fluid moves between pores. This characteristic rate is controlled by the likelihood of hopping in the probabilistic simulation. For the analytic simulation, the interpore diffusion rate is the main factor affecting intensity decay while interpore spacing has a minor influence. For the probabilistic simulation, the characteristic time between pores can be found by

$$
\lambda=\frac{\Delta t}{P},
$$

where $\lambda$ is the characteristic time, $\Delta t$ is the time step, and $P$ is the probability of hopping during that time step. Using Eq. (28) with our simulation probabilities, the characteristic times for the pores are estimated to be $\lambda_{\alpha \alpha}=2.63 \mathrm{~s}$ between $\alpha$ pores, $\lambda_{\beta \beta}=2.38$ s between $\beta$ pores, and $\lambda_{\alpha \beta}=0.71 \mathrm{~s}$ between $\alpha$ and $\beta$ pores. $\lambda$ can be simply related to the interpore diffusion coefficient by

$$
\lambda=\frac{b^{2}}{6 D_{p}},
$$

where $b$ is the interpore spacing. Our interpore diffusion rates for the analytic simulations were $D_{\alpha \alpha}=3.2$ $\times 10^{-10} \mathrm{~m}^{2} \mathrm{~s}^{-1}, \quad D_{\beta \beta}=9.3 \times 10^{-10} \mathrm{~m}^{2} \mathrm{~s}^{-1}$, and $D_{\alpha \beta}=18.0$ $\times 10^{-10} \mathrm{~m}^{2} \mathrm{~s}^{-1}$ from which we calculate $\lambda_{\alpha \alpha}=1.35 \mathrm{~s}, \lambda_{\beta \beta}$ $=3.2 \mathrm{~s}$, and $\lambda_{\alpha \beta}=0.63 \mathrm{~s}$ (Table II).

\section{E. Tortuosity}

Now that we have calculated interpore spacings and characteristic times, we can then find the tortuosity between the pores of like and unlike classes. Tortuosity is the measure of how convoluted a path is and is defined as the length of a curve divided by the distance between its end points. In the case of a pore space, we can approximate this as the free diffusion coefficient divided by the interpore diffusion coefficient

$$
T=\frac{D_{0}}{D_{p}} .
$$

This is relatively easy to calculate from the analytic model. Using the interpore diffusion coefficients of our analytic model, we obtain tortuosity values of $T_{\alpha \alpha}=7.1, T_{\beta \beta}=2.5$, and $T_{\alpha \beta}=1.3$. For the pore-hopping model, the interpore diffusion rate can be calculated from the characteristic time and the pore spacing such that

$$
D_{p}=\frac{b^{2}}{6 \lambda},
$$

where $\lambda$ is the characteristic time, and $b$ is the interpore spacing, either between like or unlike pores. This can be rewritten in terms of tortuosity to give 


$$
T=\frac{6 D_{0} \lambda}{b^{2}} .
$$

We then use our values of pore spacing and characteristic time to estimate $T_{\alpha \alpha}=11.5, T_{\beta \beta}=2.1$, and $T_{\alpha \beta}=1.8$ (Table II).

The values of tortuosities found for exchange between $\beta$ pores and between $\alpha$ and $\beta$ pores seem to agree with the calculated tortuosity from electrical conductivity. Curiously, the tortuosity between $\alpha$ pores is larger than between other two types. While tortuosity tends to be higher in general for small pores, our values appear quite high.

\section{DISCUSSION}

A noteworthy feature of our experiment is the apparently low rate of exchange of water molecules between $\alpha$ sites. The intensity for the $\alpha \alpha$ peak spreads very little beyond $20 \mu \mathrm{m}$, even at long mixing times, and we find an interpore spacing between $\alpha$ pores of around $50 \mu \mathrm{m}$ coupled with a very high tortuosity. This lack of exchange is surprising considering that there appears to be significant exchange between the $\alpha$ and $\beta$ pores at larger distances. We suggest possible solutions. First, the smaller $\alpha$ pores may be nestled in the gaps between the larger pores such that molecules need to diffuse through a larger pore before reaching another small pore, producing little direct exchange between the smaller pores. Second, the $\alpha$ pores may not be true pores. Porous media research tends to idealize pore systems into regular shapes, often spheres. However, from the x-ray CT, one can see that the pores of our sand system are more like spheres with trumpets attached. A possibility is that the $\alpha$ pores are the "trumpets" and the $\beta$ pores the spherical center of the pore. Despite the anisotropic shape of these trumpets, their isotropic distribution will produce an averaged spherical density. Therefore if this is the case, our spherical form factor assumption still holds true, though we are only finding the radius of this averaged spherical density. Further experimentation would be necessary to determine the precise dimensions of the conical pores from the apparent radius. For both situations, the fluid molecules would need to travel through a $\beta$ pore before they came to another $\alpha$ pore, leading to the high tortuosity. A third explanation is that the structure of the small pores is such that it is simply unlikely for molecules to exchange between small pores. Finally, we note that the tortuosity calculation is dependent on interpore spacing to concert a rate into a diffusion coefficient. Consequently, the abnormally high $T^{\alpha \alpha}$ may simply be an artifact of an underestimated interpore spacing. Despite this anomaly, the values of $T^{\beta \beta}$ and $T^{\alpha \beta}$ appear reasonable.

\section{CONCLUSIONS}

The propagator-resolved $T_{2}$-exchange technique represents a new approach to investigating molecular transport in porous media. The present paper outlines a first attempt to explore the potential of the method. For all our reservations expressed here, we have at least generated structural and dynamical parameters that are physically reasonable. Our technique now has the advantage of finding characteristic times between like and unlike pores. We are also able to estimate pore size without needing to know the surface relaxivity factor in order to calibrate the results. Our pore radii estimates and some of our tortuosity measurements agree well with the estimates obtained from x-ray CT while our interpore spacing values appear to underestimate the distances between pores. While our technique shows some promise in finding interpore spacing, further experimentation must be done to refine and confirm our approach. One possibility is to use flow to increase the average distance fluid molecules move, increasing the number of molecules that exchange from one pore to another. In addition, increasing the strength of our pulsed field gradients would allow us to probe a system with much smaller pores, such that most fluid molecules could diffuse from one pore over accessible time scales. Smaller pores would also mean that the fast diffusion limit assumption would be better approximated. While the estimated tortuosities between $\beta$ pores and $\alpha$ and $\beta$ pores appear reasonable, the tortuosity for exchange between $\alpha$ pores is unusually high.

The propagator-resolved $T_{2}$-exchange technique has the potential to provide a better understanding of the movement of fluid within and between pores of varying size. The pore attributes found from the experiments are useful for pore characterization and use in system modeling. The ability to compare exchange between like and unlike pore sizes could be of particular use in vuggy systems. Future work involving two-phase fluids and systems of differing wettability could give important insights to multiphase fluid flow.

\section{ACKNOWLEDGMENTS}

The authors would like to thank Y. Q. Song, R. L. Kleinberg, and M. D. Hürlimann for useful critique and E. Anderssen for helpful discussion. The authors are grateful for the $\mathrm{x}$-ray CT data acquisition and phase segmentation performed by T. J. Senden and A. P. Sheppard. The authors acknowledge the Royal Society of New Zealand Marsden fund and the Australian Research Council (Grant No. DP0558185) for financial support.
[1] R. Kleinberg and M. Horsfield, J. Magn. Reson. 88, 9 (1990).

[2] K. E. Washburn and P. T. Callaghan, Phys. Rev. Lett. 97, 175502 (2006).

[3] L. Monteilhet, J. Korb, J. Mitchell, and P. McDonald, Phys. Rev. E 74, 061404 (2006).
[4] K. Washburn and P. Callaghan, J. Magn. Reson. 186, 337 (2007).

[5] E. Stejskal and J. Tanner, J. Chem. Phys. 42, 288 (1965).

[6] P. Callaghan, Principles of Nuclear Magnetic Resonance, 1st ed. (Oxford University Press, Oxford, 1991). 
[7] M. Hurlimann, J. Magn. Reson. 131, 232 (1998).

[8] H. Carr and E. Purcell, Phys. Rev. 94, 630 (1954).

[9] S. Meiboom and D. Gill, Rev. Sci. Instrum. 29, 688 (1958).

[10] R. Wendt, P. Murphy, J. Ford, R. Bryan, and J. Burdine, Magn. Reson. Imaging 2, 527 (1985).

[11] R. Cotts, M. Hoch, and T. Sun, J. Magn. Reson. (1969-1992) 83, 252 (1989).

[12] G. Arfken, Mathematical Methods for Physicists, 3rd ed. (Academic Press, Orlando, FL, 1985).

[13] A. Tikhonov, Dokl. Akad. Nauk SSSR 151, 501 (1963).

[14] Y. Song, L. Venkataramanan, M. Hurlimann, M. Flaum, P. Frulla, and C. Straley, J. Magn. Reson. 154, 261 (2002).

[15] M. Hurlimann, L. Venkataramanan, and C. Flaum, J. Chem. Phys. 117, 10223 (2002).
[16] P. Callaghan, S. Godefroy, and B. Ryland, Magn. Reson. Imaging 21, 243 (2003).

[17] P. Callaghan and I. Furo, J. Chem. Phys. 120, 4032 (2004).

[18] A. Sheppard, R. Sok, and H. Averdunk, Physica A 339, 145 (2004).

[19] C. H. Arns, A. Sakellariou, T. J. Senden, A. P. Sheppard, R. Sok, W. V. Pinczewski, and M. A. Knackstedt, Petrophys. 46, 260 (2005).

[20] A. Sakellariou, C. H. Arns, A. P. Sheppard, R. M. Sok, H Averdunk, A. Limaye, A. C. Jones, T. J. Senden, and M. A. Knackstedt, Mater. Today 10, 44 (2007).

[21] C. Arns, M. Knackstedt, W. Pinczewski, and W. Lindquist, Geophys. Res. Lett. 28, 3361 (2001).

[22] J. Nelder and R. Mead, Comput. J. 7, 308 (1965). 